\title{
Coarse-Grained Molecular Dynamics Simulation of Perfluorosulfonic Acid Polymer in Water-Ethanol Mixtures
}

\author{
An-Tsung Kuo ${ }^{1}$, Shingo Uratal, Kazuhiro Nakabayashi, \\ Hiroyuki Watabe ${ }^{2}$, and Satoru Honmura ${ }^{2}$ \\ ${ }^{1}$ Innovative Technology Laboratories, AGC Inc., Yokohama 230-0045, Japan \\ ${ }^{2}$ Materials Integration Laboratories, AGC Inc., Yokohama 230-0045, Japan \\ ${ }^{*}$ Corresponding author. Tel: $(+)$ 81-509-014-1394. Fax: $(+)$ 81-45-374-8856.
}

Email: antsung.kuo@agc.com 


\section{Supporting Information}

(a)

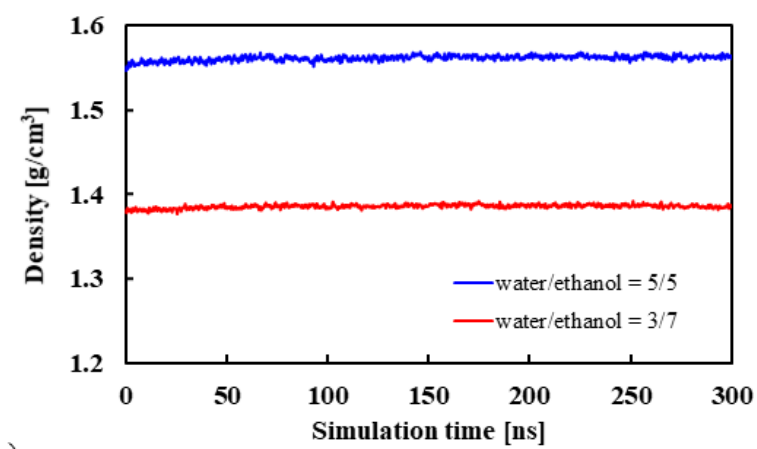

(b)

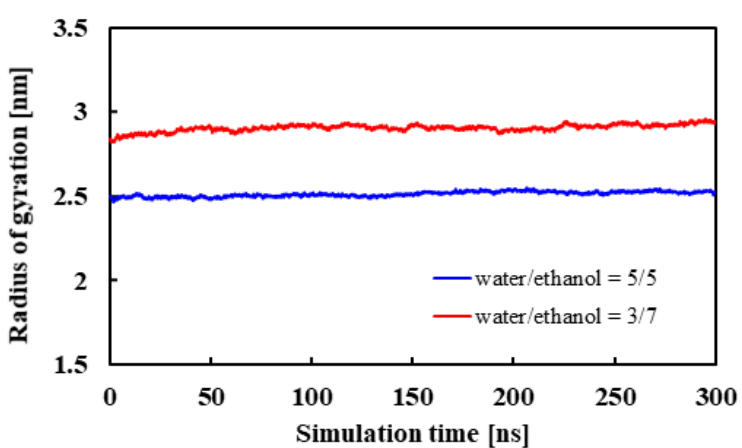

(c)

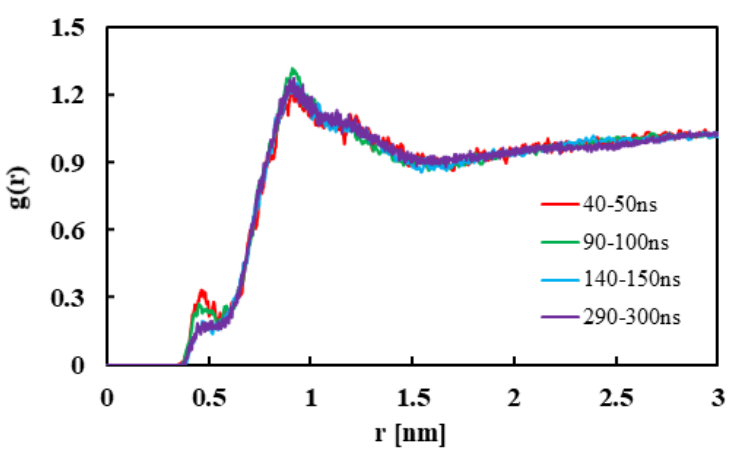

Figure S1. (a) Density and (b) radius of gyration of the polymers for the 10-mer EW 1144 PFSA membranes in 5/5 and 3/7 (w/w) water-ethanol mixtures as a function of equilibration time, and (c) radial distribution function of S-S atoms for the PFSA membrane in $3 / 7(\mathrm{w} / \mathrm{w})$ water-ethanol mixtures. 

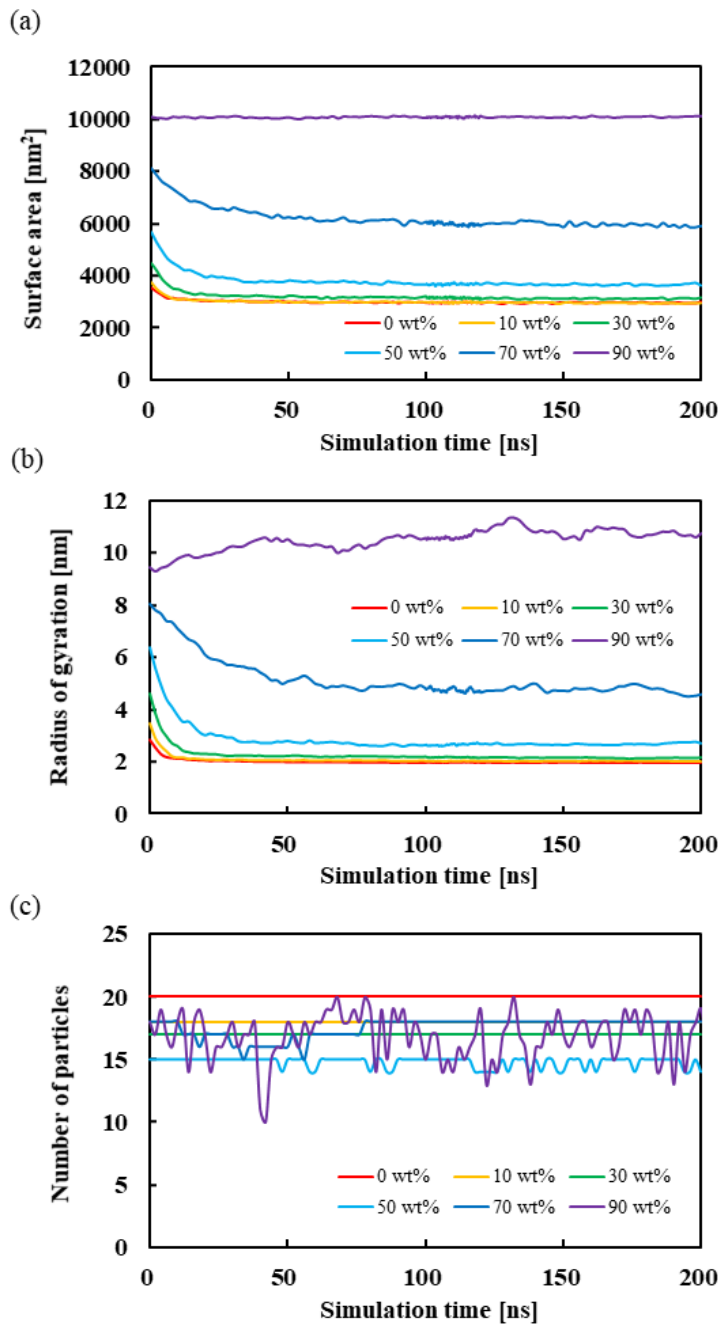

Figure S2. (a) Total solvent-accessible surface area, (b) radius of gyration of polymer, and (c) number of PFSA particles in water-ethanol mixtures as a function of equilibration time. PFSA chains are considered to be a part of the same aggregate if the distance between their backbone beads is less than $0.8 \mathrm{~nm}$, which is the position of the first minimum in the RDFs between backbone beads. At $90 \mathrm{wt} \%$ ethanol concentration, the number of particles is not convergence due to the highly solvation of PFSA chains; however, the surface area and polymer gyration radius are convergence. Except for the PFSA solution at $90 \mathrm{wt} \%$ ethanol concentration, most of PFSA particles are self-assembled by one PFSA chain, and several particles are formed by 2-3 PFSA chains. The cylindrical structures are formed after 5-40 ns equilibration run, which can be judged from the time dependency of radius of gyration. In addition, all of particles are quite stable during the simulation run. 


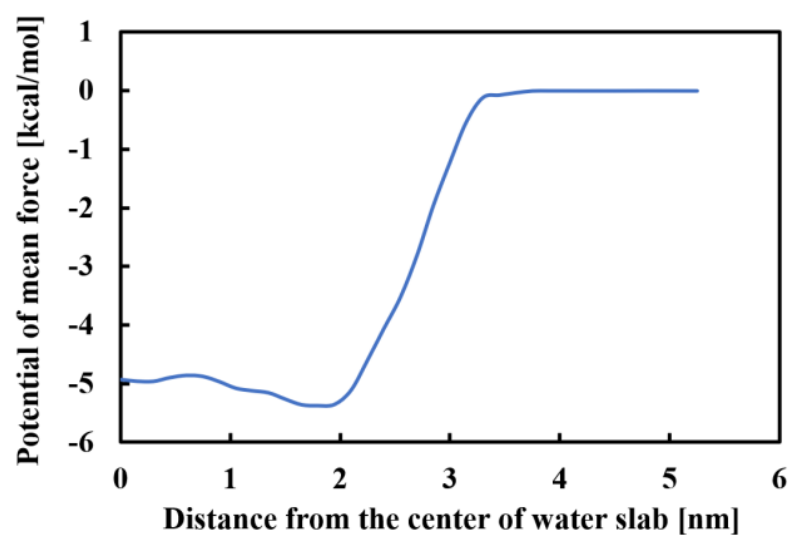

Figure S3. The free energy profile for transferring an ethanol molecule from the vacuum region to the center of the water slab. The thickness of water slab is $\sim 4.5 \mathrm{~nm}$, and the estimated hydration free energy of ethanol is $4.91 \mathrm{kcal} / \mathrm{mol}$.
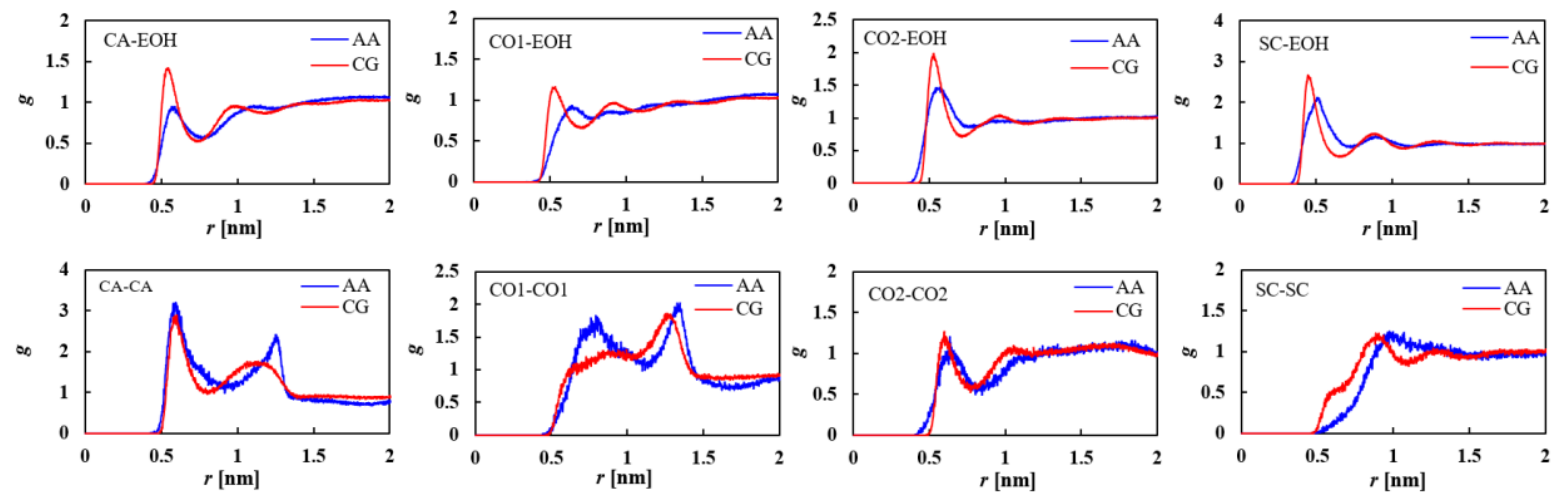

Figure S4. RDFs between the PFSA CG beads and EOH (upper panel) and RDFs between the PFSA CG beads (lower panel) for the 10-mer EW 844 membranes in $3 / 7(\mathrm{w} / \mathrm{w})$ water-ethanol mixture. 

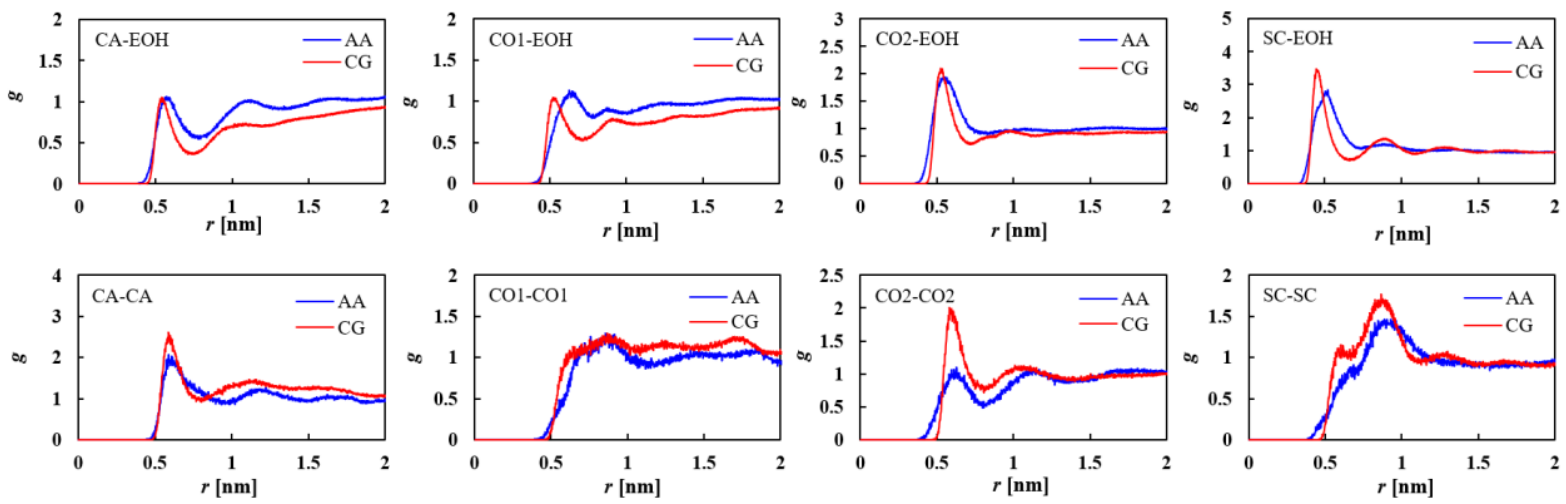

Figure S5. RDFs between the PFSA CG beads and EOH (upper panel) and RDFs between the PFSA CG beads (lower panel) for the 10-mer EW 1144 membrane in 5/5 (w/w) water-ethanol mixture.
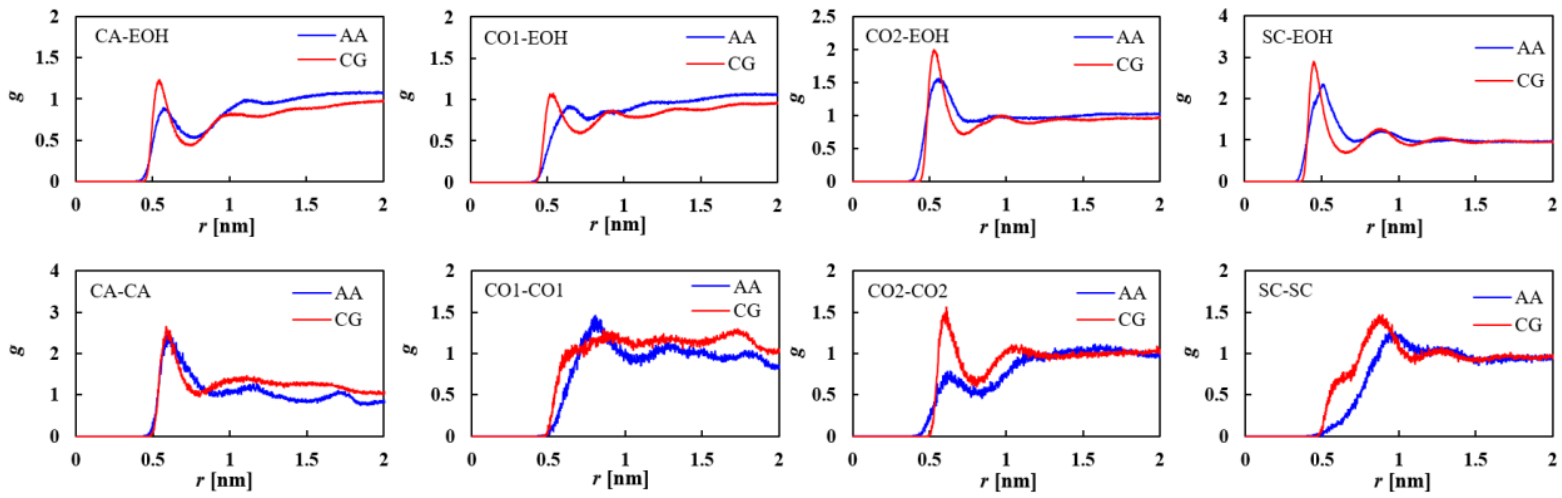

Figure S6. RDFs between the PFSA CG beads and EOH (upper panel) and RDFs between the PFSA CG beads (lower panel) for the 10-mer EW 1144 membrane in 3/7 (w/w) water-ethanol mixture. 


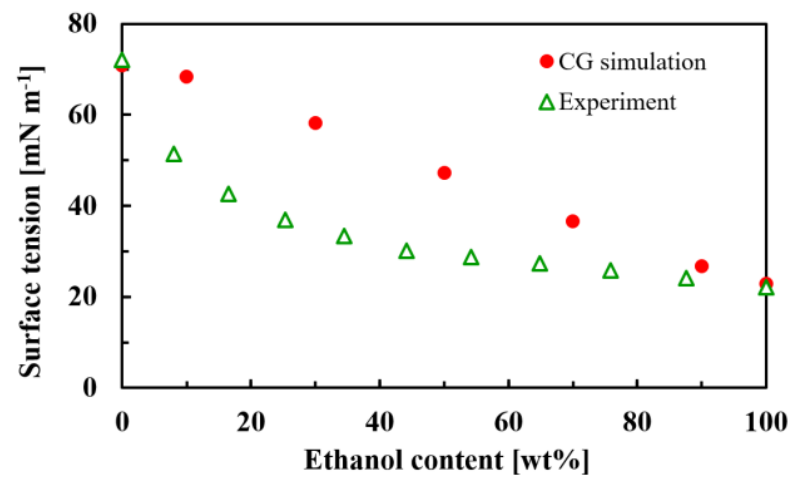

Figure S7. Surface tensions of water-ethanol mixtures as a function of ethanol content. Experiment data was taken from Ref. $1 .^{1}$

(a)

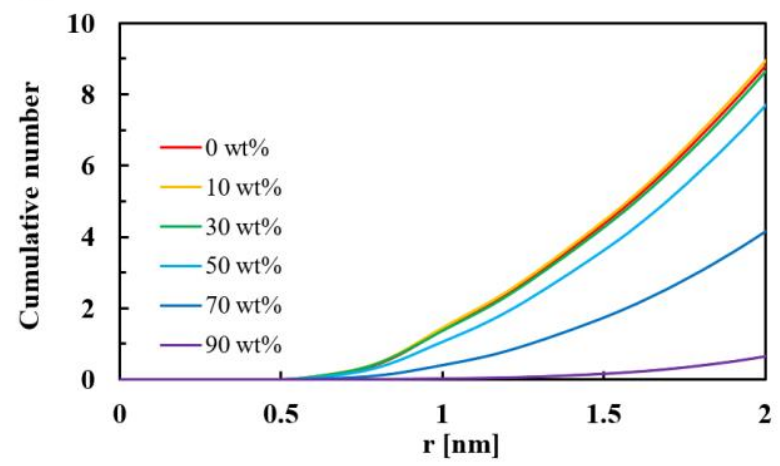

(b)

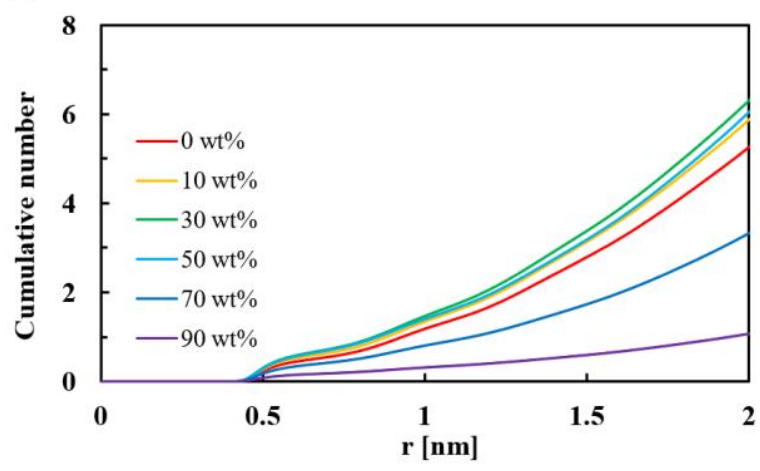

Figure S8. Cumulative number distribution functions of (a) SC-SC and (b) SC-H3O in the 60-mer EW 1144 PFSA water-ethanol mixtures with varying ethanol concentrations.

\section{REFERENCES}

(1) Khattab, I. S.; Bandarkar, F.; Fakhree, M. A. A.; Jouyban, A. J. K. J. o. C. E., Density, Viscosity, and Surface Tension of Water+Ethanol Mixtures from 293 to 323k. Korean J. Chem. Eng. 2012, 29, 812-817. 